\title{
VLT 3-5 micron spectroscopy and imaging of NGC 1068: Does the AGN hide nuclear starburst activity?^
}

\author{
O. Marco and K. J. Brooks
}

European Southern Observatory, Casilla 19001, Santiago 19, Chile

Received 8 October 2002 / Accepted 5 November 2002

\begin{abstract}
Near-infrared 3-5 micron spectroscopic and imaging observations of NGC 1068 have been obtained with the VLT at an angular resolution of $\sim 0.4 \mathrm{arcsec}$. The $[L-M]$ color index has been derived as a function of distance to the central source of the AGN and found to show significant variations. In particular, there is a blue excess of 0.2 mag in the vicinity of the dust/molecular torus. The $3.28 \mu \mathrm{m}$ polycyclic aromatic hydrocarbon (PAH) emission feature has been tentatively detected toward the central $70 \mathrm{pc}$ of the AGN, with a luminosity of $7.4 \times 10^{39} \mathrm{erg} \mathrm{s}^{-1}$. PAH emission is thought to be a strong indicator of nuclear starburst activity. Using the measured PAH 3.28 $\mu \mathrm{m}$ flux we estimate that the nuclear starburst activity in NGC 1068 may contribute up to 1 percent of the total infrared luminosity. Such a very small contribution is supported by the measured equivalent width of the PAH $3.28 \mu \mathrm{m}$ emission feature, $1.2 \mathrm{~nm}$, which is one hundred times smaller than for starburst-dominated galaxies. Absorption features at $3.4 \mu \mathrm{m}$, corresponding to carbonaceous dust have also been clearly identified yielding an optical depth value of $\tau_{3.4}=0.14$. From this we derive an extinction of $A_{V}=28 \mathrm{mag}$ toward the central source. The temperature and mass of the hot dust present in the inner 200 parsecs of the central source are estimated from the $L$ - and $M$-band photometry. For an average temperature of $T_{\text {grain }} \sim 475 \mathrm{~K}$, we find $M_{\text {hot dust }} \sim 0.6 M_{\odot}$. Barely detected and at $L$ band only, the contribution of the kilo-parsec-scale ring of star formation is found to be negligible in the $3-5 \mu \mathrm{m}$ domain.
\end{abstract}

Key words. galaxies: individual: NGC 1068 - galaxies: Seyfert - galaxies: nuclei - infrared: galaxies - galaxies: starburst

\section{Introduction}

Polycyclic aromatic hydrocarbon (PAH) emission features are well-established tracers of star-formation activity and are now becoming an important tool for identifying the presence of any nuclear starburst activity in active-galactic nuclei (AGN). Such activity is thought to be a consequence of a dust/molecular torus surrounding the accretion disk of an AGN (Cid Fernandes $\&$ Terlevich 1995). The detection of $3.4 \mu \mathrm{m}$ carbonaceous dust and $9.7 \mu \mathrm{m}$ silicate absorption features toward the central engines of AGN provide further evidence for the existence of a dust/molecular torus. These features are now being used to identify whether AGN are obscured or not (Imanishi 2000a).

Since X-ray photons are very efficient in the destruction of PAHs they are not expected to survive near an AGN. Such an assumption is based on the results by Voit (1992) who studied the destruction and survival of PAHs in AGN. His work was mainly based on previous mid-infrared observations by Roche et al. (1991) which showed that starburst galaxies always show prominent PAH 8.7 and $11.3 \mu \mathrm{m}$ emission features, whereas the spectra of AGN are usually featureless. Voit (1992) and Miles et al. (1994) argued that if PAH emission is detected in an AGN, the molecules must be shielded from the central X-ray source by a substantial column density of X-ray

\footnotetext{
Send offprint requests to: O. Marco, e-mail: omarco@eso.org

$\star$ Based on observations collected at the European Southern Observatory, Paranal, Chile, (ESO Programme 67.B-0332).
}

absorbing material $\left(N_{\mathrm{H}} \geq 10^{24} \mathrm{~cm}^{-2}\right)$. In this case, the PAHs can not be excited directly by the central engine and must therefore be excited by local soft UV emission, coming from localized starburst activity.

In cases where localized nuclear starburst activity is found, it is of interest to know the contribution to the overall luminosity, with respect to the contribution from AGN activity. With such information it would then be possible to determine if any relations exist, (for instance if the brightest AGN also host the brightest compact nuclear starbursts), and to build an evolutionary scenario.

NGC 1068 is a bright, nearby Seyfert 2 active galaxy and considered the best prototype for testing AGN models (Antonucci 1993; Krolik 1999). The dust/molecular torus surrounding the AGN has been tentatively observed using adaptive-optic systems (Lacombe et al. 1998) in the nearinfrared by Marco \& Alloin (2000) and Rouan et al. (1998). Both sets of results show disk-like structures extending eastwest (a few tens of parsecs) from the central peak of infrared emission. Spectroscopic observations by Alloin et al. (2001) have traced $\mathrm{H}_{2}$ 1- $0 \mathrm{~S}(1) 2.12 \mu \mathrm{m}$ emission also along an eastwest direction and identified two peak emission knots, both located at a distance of about $70 \mathrm{pc}$ from the central engine. The authors find a velocity difference of $140 \mathrm{~km} \mathrm{~s}^{-1}$ between the two knots, suggesting a central enclosed mass of $10^{8} M_{\odot}$.

NGC 1068 is known to host a ring of star formation at a distance of $1 \mathrm{kpc}$ from the central source of the AGN 
(Davies et al. 1998). The star formation ring is seen face on and no other extended star-formation region in the foreground of the NLR region has been detected so far.

We present new results for NGC 1068 based on observations involving imaging and spectroscopy in the near-infrared (3-5 $\mu \mathrm{m})$ with ISAAC at the VLT. The observations yield sufficient sensitivity to study further the environment in the vicinity of the AGN and to determine whether or not starburst activity is present. Assuming $H_{0}=75 \mathrm{~km} \mathrm{~s}^{-1} \mathrm{Mpc}^{-1}$, NGC 1068 with $z=0.0038$ (Bottinelli et al. 1990), is at a distance of $14.4 \mathrm{Mpc}$ and 1 arcsec corresponds to $72 \mathrm{pc}$.

\section{Observations and data reduction}

Observations have been made with ISAAC on the VLT UT1 telescope, at the ESO Paranal Observatory, in service mode. The long wave camera $(\lambda>3.0 \mu \mathrm{m})$ was utilised which is equipped with a SBRC Aladdin $1024 \times 1024$ pixel InSb array. The imaging part was taken on the night of 14-15 August 2001 (UT), and the spectroscopic part on the night of 17-18 August 2001 (UT), both under photometric conditions. Calibration observations (standard stars, flat fields, dark current and arcs) were obtained as part of the ESO calibration plan.

\subsection{Imaging}

NGC 1068 was observed at an airmass of $\sim 1.3$ using the broadband filter $L^{\prime}(3.78 \pm 0.29 \mu \mathrm{m})$ and the narrow-band filter $M^{\prime}$ $(4.66 \pm 0.05 \mu \mathrm{m})$, with a pixel scale of 0.071 arcsec, giving a field of view of $72 \times 72 \mathrm{arcsec}^{2}$.

To account for the high sky background, the observations were made in chopping mode, using a chopping throw of 15 arcsec. In the respective $L^{\prime}$ - and $M^{\prime}$-bands, 20 and 16 exposures of $30 \mathrm{~s}$ were taken, resulting in an equivalent exposure times of 600 and $480 \mathrm{~s}$. The limiting magnitude for an extended source with a $3 \sigma$ detection is 13.1 in $L^{\prime}$ band and 10.5 in $M^{\prime}$ band.

The individual raw images were first corrected for flatfield effects and dead pixels and then combined using telescope offset values that were refined with a cross-correlation method. The zero points were determined using the standard star HD205772 (A3-type) with a similar airmass. The results are $22.44 \pm 0.01$ in $L^{\prime}$ band and $19.63 \pm 0.01$ in $M^{\prime}$ band

The $L^{\prime}$ - and $M^{\prime}$ - band images have been combined to produce a $\left[L^{\prime}-M^{\prime}\right]$ color map. They have been re-centered so that their central peaks are coincident. This assumption is based on a previous study by Marco \& Alloin (2000) using an adaptive optics system.

\subsection{Spectroscopy}

NGC 1068 was observed at an airmass of $\sim 1.1$, using a slit of 1 arcsec in the low-resolution mode: pixel scale of 0.146 arcsec/pixel, spectral resolution of 360 and wavelength coverage of 3-4.2 $\mu \mathrm{m}$. The slit length was $120 \mathrm{arcsec}$, allowing the use of a chopping throw of 15 arcsec for the sky-background determination.

The raw images were first corrected for flat-field effects and dead pixels, then sky subtracted and wavelength calibrated
Table 1. Photometric results for NGC 1068.

\begin{tabular}{lccccc}
\hline \hline & \multicolumn{5}{c}{ aperture radius (pc) } \\
& 25 & 50 & 100 & 200 & 500 \\
\hline magnitude $L^{\prime}$ & 5.62 & 4.88 & 4.61 & 4.53 & 4.48 \\
magnitude $M^{\prime}$ & 4.52 & 3.73 & 3.44 & 3.37 & 3.35 \\
\hline
\end{tabular}

Note.- The fluxes have been computed on raw (non-deconvolved) images.

using spectroscopic arcs and flats. The resultant images were combined and then a single spectrum was extracted (using a band of 8 pixels along the slit direction which corresponds to $\sim 1^{\prime \prime}$ ). The spectroscopic standard, BS88 (G2V-type), was observed under similar conditions and used to remove the atmospheric features and flux calibrate the spectrum.

\section{Results}

\subsection{Images}

The $L^{\prime}$ - and $M^{\prime}$-band images show a bright, unresolved central peak of emission surrounded by extended emission up to a distance of $500 \mathrm{pc}$ from the peak. $90 \%$ of the flux is contained in a region less than $100 \mathrm{pc}$ away from the central peak. We present in Fig. 1 the reduced image obtained with the $L^{\prime}$-band filter. The $F W H M$ measured on the standard star observed just after the object is $\sim 0.4$ arcsec, which corresponds to $28 \mathrm{pc}$ at the distance of NGC 1068; the FWHM on the extended object is $\sim 0.65$ arcsec.

The well-known starburst ring is barely detected in the $L^{\prime}$ band image (1- $\sigma$ detection only) and not detected at all in the $M^{\prime}$ band image. On the presented $L^{\prime}$ image, the outer regions of emission correspond to the star-forming knots from Davies et al. (1998): A, B and D to the north-east and F, G and H to the south-west. According to these authors, these regions are the most massive with a mass of $\sim 10^{6} M \odot$ and an age of $\sim 6 \mathrm{Myr}$. The non-detection in $M^{\prime}$-band may be a consequence of the difference in limiting magnitude $(\sim 2.5)$ between these bands. For a typical dust extinction in starbursts of $A_{v}=2-8 \mathrm{mag}$ (Gordon et al. 2000), the extinction correction factor in the $L^{\prime}$ band would be $\sim 0.3$, while $\sim 0.1$ in the $M^{\prime}$ bands. The limiting magnitude in $L^{\prime}$ corresponds to $\sim 2 \mathrm{mJy}$ (Sect. 2.1), while the total flux of NGC 1068 is $\sim 4 \mathrm{Jy}$ in the $L^{\prime}$ band and $\sim 10 \mathrm{Jy}$ in the $M^{\prime}$ band (Table 1). Therefore the contribution of the circumnuclear starburst ring in the 3-5 $\mu \mathrm{m}$ domain to the total infrared luminosity of NGC 1068 is negligible.

\subsection{Photometry and color index}

The high angular resolution of the images is sufficient to perform circular aperture photometry. Table 1 lists the magnitude as a function of distance from the central source. The color analysis does not include the kiloparsec starburst ring, located farther away and detected only on in the $L^{\prime}$ band. We find $\left[L^{\prime}-M^{\prime}\right]=1.13 \pm 0.02 \mathrm{mag}$ for the whole region of infrared emission (1 kpc in diameter). Previously published photometry by Marco \& Alloin (2000) give a color of $1.6 \pm 0.4$ inside an aperture of $42 \mathrm{pc}$; Alonso-Herrero et al. (2001) found $\left[L^{\prime}-M^{\prime}\right]=1.3 \pm 0.1 \mathrm{mag}$. The flux density has been computed for the $L^{\prime}$ and $M^{\prime}$ bands as a function of dis- 


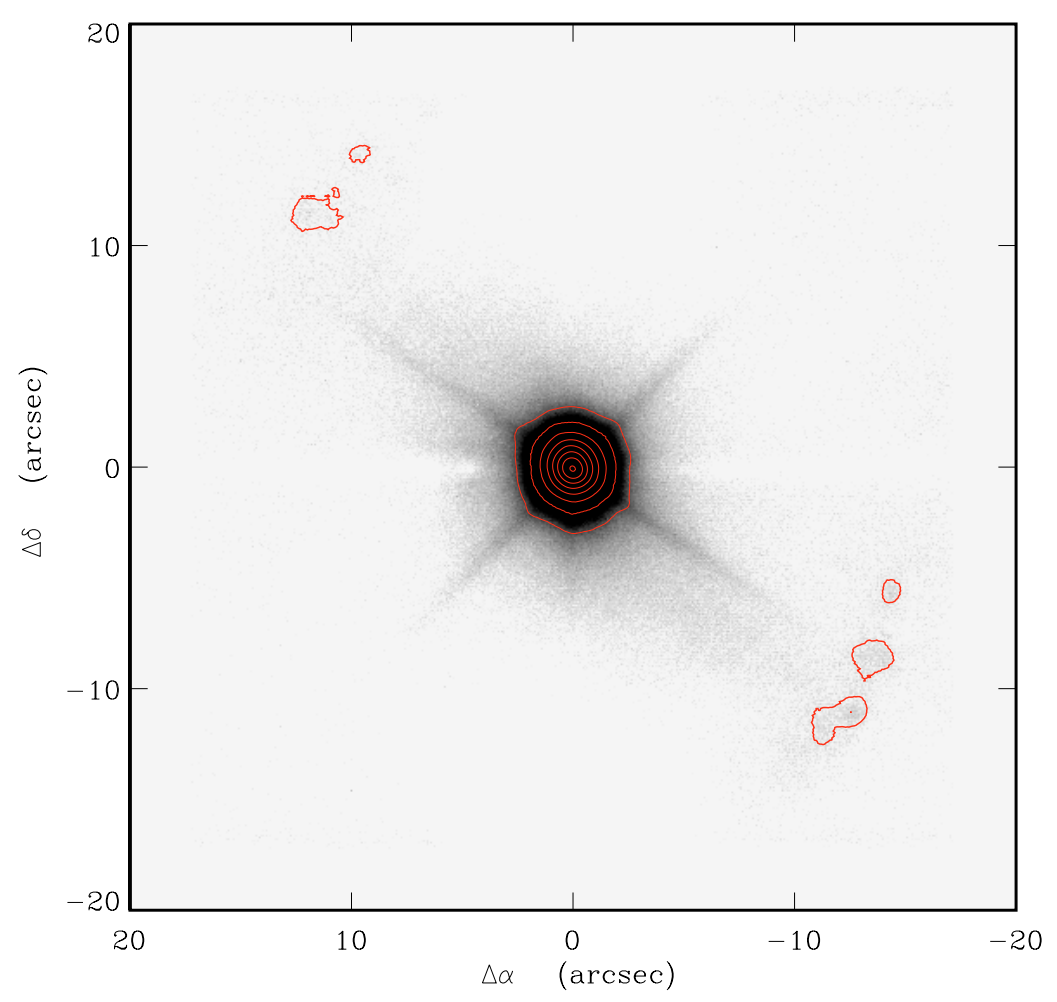

Fig. 1. The $L^{\prime}$-band image of NGC 1068 presented in a log grey-scale. The contours delimiting the circumnuclear starburst knots correspond to the $1 \sigma$ detection limit. North is up and east to the left.

Table 2. Spectroscopic results for NGC 1068.

\begin{tabular}{lcccc}
\hline \hline \multirow{2}{*}{ Line } & \multicolumn{2}{c}{ Luminosity } & \multicolumn{2}{c}{ Rest EW } \\
& $\begin{array}{c}\text { × } \\
\times 10^{39} \mathrm{ergs} \mathrm{s}^{-1}\end{array}$ & \multicolumn{2}{c}{$\times 1 \mathrm{~nm}$} \\
& Our data & Literature & Our data & Literature \\
\hline PAH 3.28 $\mu \mathrm{m}$ & 7.4 & $<27$ & 1.2 & $<1.2$ \\
{$[\mathrm{Mg} \mathrm{VIII}] 3.028 \mu \mathrm{m}$} & 31 & 27 & 5 & \\
\hline
\end{tabular}

Note.- PAH $3.28 \mu \mathrm{m}$ values from Imanishi (2002) and [Mg VIII] $3.028 \mu \mathrm{m}$ values from Alexander et al. (2000).

tance from the central source (Fig. 2), using circular rings centered on the central peak of emission. The result can be fitted by a power law $F(r) \propto r^{-1.4}$.

Using the $L^{\prime}$ and $M^{\prime}$ flux densities we also determined the color index as a function of the distance from the central source (Fig. 3). The analysis reveals 3 distinct regions: a central region $(r<30 \mathrm{pc})$, with $\left[L^{\prime}-M^{\prime}\right]=1.1 \mathrm{mag}$; an intermediate region $(50<r<130 \mathrm{pc})$, with $\left[L^{\prime}-M^{\prime}\right]=1.1-1.3 \mathrm{mag}$ and an external region $(r>150 \mathrm{pc})$, with $\left[L^{\prime}-M^{\prime}\right]<1.1 \mathrm{mag}$.

Typical $\left[L^{\prime}-M^{\prime}\right]$ color indices of AGN and starburst galaxies have been studied in detail by Imanishi (2001) and found to be: $1.0 \pm 0.1$ for QSO; $\sim 0.8$ for Type $1 ; \sim 0.4$ for starbursts and $\sim 1.0$ for Type 2 .

\subsection{Spectroscopy}

The slit has been centered on the brightest peak of emission previously detected in $K$ band and oriented North-South. This peak corresponds to the location of the hidden central engine and the peak of emission at 2.2, 3.4, 4.8 and 10-12.4 $\mu \mathrm{m}$ (Marco et al. 1997; Marco \& Alloin 2000; Braatz et al. 1993; Tomono et al. 2001). The slit width of 1 arcsec corresponds to

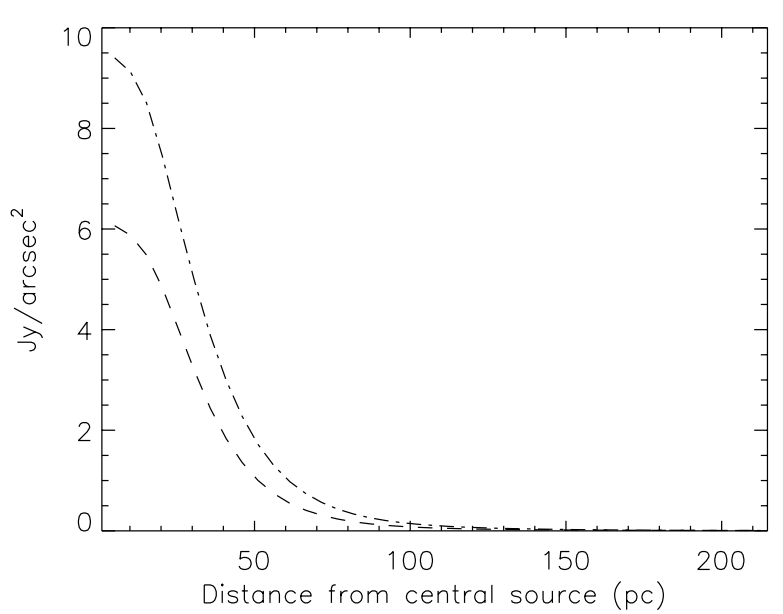

Fig. 2. Flux density as a function of the distance to the central source. Dashed line is for $L^{\prime}$ band and dashed-dotted line is for $M^{\prime}$ band.

$70 \mathrm{pc}$, and the spectrum was extracted using an aperture along the slit direction corresponding to $\sim 1^{\prime \prime}$. Thus it is fair to assume that the light analysed in our spectra comes from the central $70 \mathrm{pc}$ (diameter) of the AGN. This includes the hidden central 


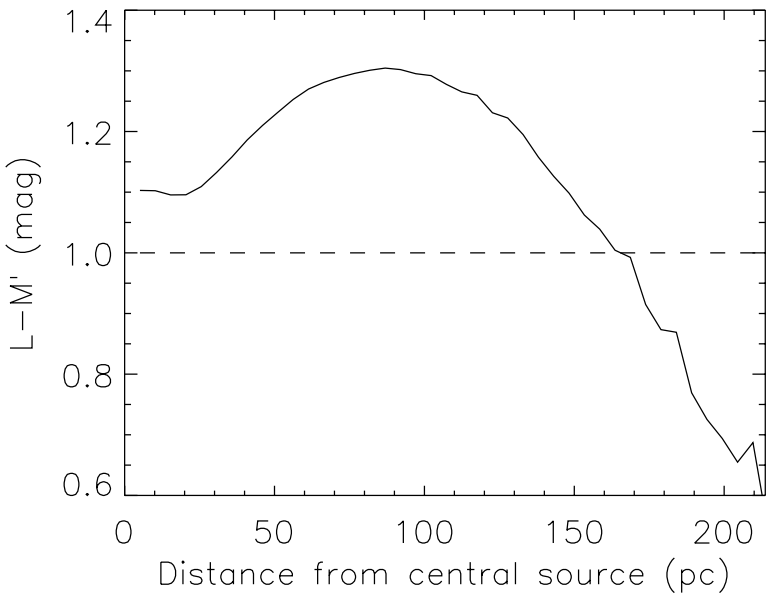

Fig. 3. $L^{\prime}-M^{\prime}$ color index of NGC 1068 as a function of the distance to the central source. Colors have been derived from the flux density distribution shown in Fig. 2.

engine and the dust/molecular torus and excludes the starburst ring.

The ISAAC spectrum is presented in Fig. 4. An absorption feature centred near $3.4 \mu \mathrm{m}$ corresponding to carbonaceous dust is clearly detected as well as emission line from [Mg VIII] $3.028 \mu \mathrm{m}$ and PAH $3.28 \mu \mathrm{m}$ (albeit a low signal to noise ratio for the latter).

The spectrum shows a very steep continuum that can be fitted by a power-law $F_{v} \propto v^{-\alpha}$ with a slope $\alpha=4.0$ (in the $L^{\prime}$ band). This is much steeper than power-law indices observed in non-thermal sources and is attributed to optically thin dust emission. Thatte et al. (1997) find a value of $\alpha=4.9$ from a $K$-band spectrum of NGC 1068 within a radius of 0.5 arcsec centered on the $K$-band emission peak, which corresponds to our slit position and size.

The measured emission fluxes and the rest-frame equivalent widths (EW) for the PAH $3.28 \mu \mathrm{m}$ and [Mg VIII] $3.028 \mu \mathrm{m}$ lines are presented in Table 2 and compared with previously published values. For the bright [Mg VIII] $3.028 \mu \mathrm{m}$ line we measure a flux of $1.3 \times 10^{-15} \mathrm{~W} \mathrm{~m}^{-2}$, in agreement with the value of $1.1 \times 10^{-15} \mathrm{~W} \mathrm{~m}^{-2}$ previously published by Alexander et al. (2000). For the PAH $3.28 \mu \mathrm{m}$ line, we find a value of $3.0 \times 10^{-16} \mathrm{~W} \mathrm{~m}^{-2}$, in agreement with the upper limit of $<9 \times$ $10^{-16} \mathrm{~W} \mathrm{~m}^{-2}$ published by Imanishi (2002).

\section{Discussion}

\subsection{Absorption at $3.4 \mu \mathrm{m}$ and AGN activity}

NGC 1068 is known to be highly dust obscured. X-ray spectroscopic observations by Matt et al. (1997) have shown it is a Compton thick source with $N_{\mathrm{H}}>10^{24} \mathrm{~cm}^{-2}$. The detection of the carbonaceous dust absorption feature centred near $3.4 \mu \mathrm{m}$ is a confirmation of the obscuration of the AGN by molecular material. Indeed, Tomono et al. (2001) presented 0.1 arcsec images of NGC 1068 in the mid-infrared, showing the silicate absorption feature at $9.7 \mu \mathrm{m}$ towards the central emission peak $\left(\tau_{9.7}=0.7\right.$ within an aperture size of $\left.30 \mathrm{pc}\right)$. They conclude that the silicate absorption feature originates in dusty regions close $(<10 \mathrm{pc})$ to the central source.
Using our $L$-band spectra, we find an optical depth of the $3.4 \mu \mathrm{m}$ carbonaceous dust absorption of $\tau_{3.4}=0.14$, within an aperture of $\sim 1$ ". This value is larger than previous published values, measured using larger apertures: Bridger et al. (1994) reported $\tau_{3.4}=0.09$ within an aperture of $\sim 3.1 \times 3.1^{\prime \prime}$ and Imanishi et al. (1997) reported $\tau_{3.4}=0.12$ within an aperture of $\sim 3.8 \times 3.8^{\prime \prime}$. The larger value of $\tau_{3.4}$ reported here could result from the smaller aperture used, since the contamination from weakly-obscured emission to the observed 3-4 $\mu \mathrm{m}$ flux is smaller. For a typical galactic interstellar medium, Pendleton et al. (1994) give $\tau_{3.4} / A_{V} \simeq 0.005$. Based on this ratio we derive $A_{V}=28 \mathrm{mag}$, in agreement with the published $A_{V}=30 \mathrm{mag}$ from Marco \& Alloin (2000) and the value of $A_{V}=18-30 \mathrm{mag}$ published by Imanishi et al. (1997).

Using the results from Tomono et al. (2001) we can derive the ratio $\tau_{3.4 \mu \mathrm{m}} / \tau_{9.7 \mu \mathrm{m}} \sim 0.2$, which is about 3 times higher than that toward the Galactic center. One explanation for such a high obscuration could be the presence of a temperature gradient in the vicinity of the central engine (Imanishi 2000b). In fact, such a temperature gradient has been observed in NGC 1068 using adaptive optics imaging (Marco et al. 2000), although at a larger scale: from $1500 \mathrm{~K}$ at the location of the infrared emission peak to $\sim 500 \mathrm{~K}$ at a distance of $20 \mathrm{pc}$ and a few hundreds of Kelvin at $100 \mathrm{pc}$.

\subsection{PAH 3.28 $\mu \mathrm{m}$ emission and starburst activity}

The weak PAH $3.28 \mu \mathrm{m}$ emission line detected in our spectra (Fig. 4) confirms earlier reports made by Marco (1997) and Tomono et al. (2001). Imaging detections of PAH $3.28 \mu \mathrm{m}$ emission using adaptive optics (albeit with uncalibrated data) had been previously reported by Marco (1997) and the emission was found to coincide with the peak $L^{\prime}$-band emission. Tomono et al. (2001) presented the SED of the central $30 \mathrm{pc}$ (diameter) region of NGC 1068 in the mid-infrared. The flux at $11.7 \mu \mathrm{m}$ was found to be larger than that at $12.3 \mu \mathrm{m}$, suggesting the presence of PAH emission. Moreover, the SED for the central $20 \mathrm{pc}$ (diameter) region shows two peaks of emission at 7.7 and $8.7 \mu \mathrm{m}$, a signature of PAH emission. It seems that the PAH emission detected toward NGC 1068 at 3.28, 7.7, 8.7 and $11.7 \mu \mathrm{m}$ originates from the immediate $(r<30 \mathrm{pc})$ surroundings of the dust/molecular torus. The PAH molecules must be sheltered from the X-ray and UV emission from the central source by the material of the dust/molecular torus.

The infrared luminosity of NGC 1068 is $L_{\mathrm{IR}}=2 \times 10^{11} L \odot$. From our spectra, we find $f_{3.28 \mathrm{PAH}}=3.0 \times 10^{-16} \mathrm{~W} \mathrm{~m}^{-2}$ which corresponds to $L_{3.28 \mathrm{PAH}}=7.4 \times 10^{39} \mathrm{erg} \mathrm{s}^{-1}$ at the distance of NGC 1068. The typical luminosity ratio for compact nuclear starbursts in Seyfert 2 has been published by Imanishi (2002) and Mouri et al. (1990): it is $L_{3.28 \mathrm{PAH}} / L_{\mathrm{IR}}$ starburst $=10^{-3}$. We can infer from this ratio that the infrared contribution from a putative nuclear starburst is $\sim 7.4 \times 10^{42} \mathrm{erg} \mathrm{s}^{-1}$ or $1 \%$ of the total infrared luminosity of NGC 1068.

The equivalent width of the PAH $3.28 \mu \mathrm{m}$ emission feature is found to be $1.2 \mathrm{~nm}$, about a hundred times less than the typical value for starburst dominated galaxies given by Imanishi (2002). This is an expected confirmation that, although present, the compact nuclear starburst activity within NGC 1068 has a 


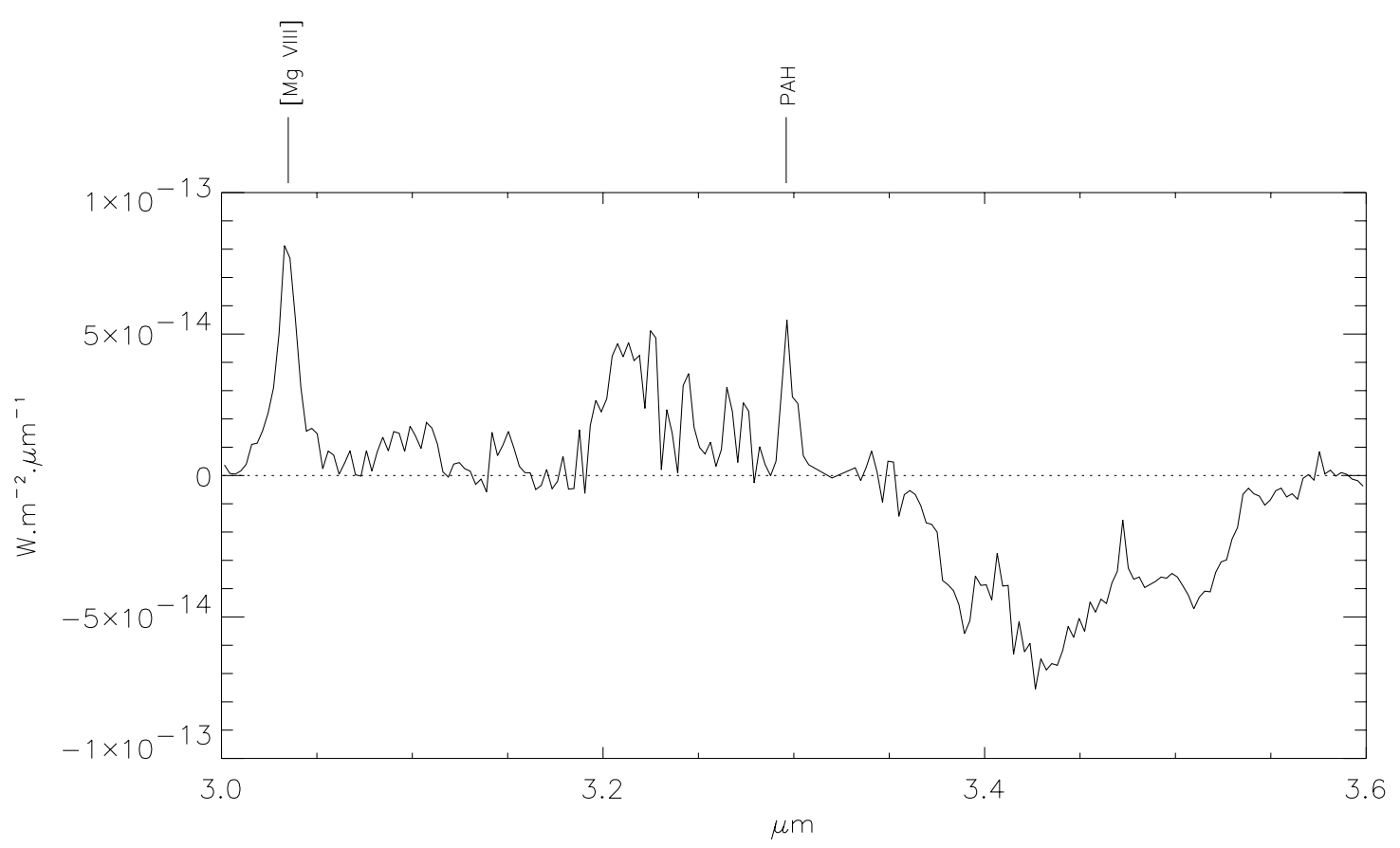

Fig. 4. The $L$ band spectrum of NGC 1068 in the 3-3.6 $\mu$ m range. The continuum level has been removed for clarity.

negligible contribution to the total infrared luminosity when compared to the contribution from AGN activity.

\subsection{Color index variation}

As previously mentioned, the $\left[L^{\prime}-M^{\prime}\right]$ local color index presented in Fig. 3 shows significant variations with distance from the central engine. Within the inner $30 \mathrm{pc}$ of the AGN central region, the color index appears to have a blue excess of $0.2 \mathrm{mag}$ when compared to that at $80 \mathrm{pc}$ away. This excess could be explained by the presence of the compact nuclear starburst discussed in Sect. 4.2. A change of $0.2 \mathrm{mag}$ at $L^{\prime}$-band corresponds to $\sim 6 \times 10^{42} \mathrm{erg} \mathrm{s}^{-1}$ which is consistent with the estimated infrared contribution of the starburst activity (Sect. 4.2).

One possible explanation for the variation of the color index with the distance to the central source of the AGN could be local extinction. The $L^{\prime}$ band is sensitive to higher-temperature dust than the $M^{\prime}$ band (typically $770 \mathrm{~K}$ for $L^{\prime}$ band and $620 \mathrm{~K}$ for $M^{\prime}$ band). The hottest grains are located in the inner part of the dust/molecular torus, close the central heating source, while the warmest grains are found farther away, where the dust density is lower. This will result in a higher flux attenuation in the $L^{\prime}$ band than in the $M^{\prime}$ band. Using $A_{V}=28$ (Sect. 4.1), $A_{L} / A_{V}=0.058, A_{M} / A_{V}=0.023$ (Cardelli et al. 1989) we find $A_{L} \approx 1.6 \mathrm{mag}$ while $A_{M} \approx 0.6 \mathrm{mag}$, producing a redder [ $L^{\prime}-$ $M^{\prime}$ ] color index.

To compute the distance at which a temperature of $770 \mathrm{~K}$ is reached, we rely on Barvainis (1987). The temperature of the dust grains follows a power law: $T_{\text {grain }}(r)=$ $1650 L_{\mathrm{UV}, 46}^{0.18} r^{-0.36} \mathrm{~K}(1)$, where $L_{\mathrm{UV}, 46}$ is the UV luminosity in units of $10^{46} \mathrm{erg} \mathrm{s}^{-1}$ and $r$ the radial distance in parsecs. Assuming $L_{\mathrm{UV}}=4 \times 10^{45} \mathrm{erg} \mathrm{s}^{-1}$ in the case of NGC 1068 (Marco \& Alloin 2000) we find $r \approx 6 \mathrm{pc}$ for $T_{\text {grain }}=770 \mathrm{~K}$, a distance well under our spatial resolution. Therefore, since the very hot dust is located very near to the central source of the
AGN, the observed color index variations can not be attributed to local extinction of the infrared emission by the dust.

\subsection{Dust temperature and mass}

From Eq. (1), we derive that at $100 \mathrm{pc}$ from the central source, the temperature of the grains is $\sim 270 \mathrm{~K}$, decreasing down to $\sim 200 \mathrm{~K}$ at $200 \mathrm{pc}$. Using a grey-body emission law, we derive the dust temperature corresponding to the mean color index of the whole region of infrared emission, $\left[L^{\prime}-M^{\prime}\right]=1.13$. It is found to be $T_{\text {grain }} \sim 475 \mathrm{~K}$, showing that the emission from the hottest grains dominates.

Following Barvainis (1987), the infrared spectral luminosity of an individual grain of dust is $L_{v, \mathrm{IR}}^{\text {grain }}=$ $4 \pi a^{2} \pi q_{\mathrm{IR}} v^{\gamma} B_{v}\left(T_{\text {grain }}\right)$ where $a$ is the radius of the grains ( $\sim 0.05 \mu \mathrm{m}$ for graphite), $q_{\mathrm{IR}}=1.4 \times 10^{-24}$ and $\gamma=1-2$, the index of the grey-body law. Using the measured $L^{\prime}$ - and $M^{\prime}$ band luminosities and a temperature $T_{\text {grain }} \sim 475 \mathrm{~K}$, we can deduce the mass of hot dust present in the inner $200 \mathrm{pc}$ region around the central source. With a grain density $\rho=2.26 \mathrm{~g} \mathrm{~cm}^{-3}$ (Barvainis 1987), the mass of hot dust grains $\left(T_{\text {grain }} \sim 475 \mathrm{~K}\right.$ ) is found to be $M_{\text {hot dust }} \sim 0.6 M_{\odot}$. Most of the dust present in the ISM must be cool $(\leq 100 \mathrm{~K})$ and is not detected in the near-infrared.

The mass of hot dust grains in the case of NGC 1068 is higher than the value of $\sim 0.05 M_{\odot}$ found for NGC 7469 by Marco \& Alloin (1998) and of $\sim 0.02 M_{\odot}$ in the case of Fairall 9, published by Clavel et al. (1989). However, the mass of hot dust depends on the grain composition and grain size distribution. The intense radiation from the central source of the AGN, the high temperature and density in the central 70 pc of the AGN could modify the dust grains composition and sizes. For instance, the sublimation temperature of silicate grains $(\sim 1000 \mathrm{~K})$ is lower than for graphite grains $(\sim 1500 \mathrm{~K})$. A larger size of the dust grains compared to typical galactic interstellar medium values 
would change the $\left[L^{\prime}-M^{\prime}\right]$ color index to smaller values, the smallest grains being destroyed by the radiation from the central source or coagulated into larger grains. Therefore, the mass of hot dust in the $200 \mathrm{pc}$ region around the central source remains uncertain.

\section{Conclusion}

Combined with previously published results of NGC 1068, the ISAAC-VLT near-infrared $(3-5 \mu \mathrm{m})$ observations presented here allow us to build a more complete sketch of this AGN:

1. The central engine is embedded in a parsec-scale dust/molecular torus, with dust at a temperature close to the evaporation temperature (1000-1500 K), and concentrated in a bright, unresolved central peak of emission. The ratio of $3.4 \mu \mathrm{m}$ carbonaceous dust and $9.7 \mu \mathrm{m}$ silicate absorption features toward the central engine $\left(\tau_{3.4 \mu \mathrm{m}} / \tau_{9.7 \mu \mathrm{m}}\right)$ is $\sim 0.2$, which is about 3 times higher than toward the Galactic center.

2. In the $70 \mathrm{pc}$ diameter central region there is evidence for very weak nuclear starburst activity via the tentative detections of PAH emission features. If this is the case then the starburst activity, when compared to the AGN, has a negligible contribution to the total infrared luminosity (up to $1 \%$ ).

3. Hot dust is present all around the central engine, up to a distance of $500 \mathrm{pc}$. The dust temperature and mass have been estimated using the $\left[L^{\prime}-M^{\prime}\right]$ color index, with a mass of hot dust grains $\left(T_{\text {grain }} \sim 475 \mathrm{~K}\right.$ ) found to be $M_{\text {hot dust }} \sim 0.6 M_{\odot}$.

4. At a kilo-parsec distance, there is a ring of star-forming regions whose infrared counterpart is detected in the $L^{\prime}$ band only and found to be negligible (less than $0.1 \%$ ) in the $3-$ $5 \mu \mathrm{m}$ domain.

The detection of weak PAH $3.28 \mu \mathrm{m}$ emission towards the nucleus of NGC 1068 needs to be confirmed using high-resolution spectroscopy to unambiguously identify PAH $3.28 \mu \mathrm{m}$ features and high-resolution narrow-band imaging so that the spatial distribution is clear. Beyond that, more AGNs, not only Seyfert 2, should be observed in order to assess the frequency of nuclear starburst events in the vicinity of AGNs and their energetic importance. An evolutionary process in the AGN unification schemes, including temporary compact nuclear starbursts events may then be built. Ideally observations to image the PAH $3.28 \mu \mathrm{m}$ emission should be made on an adaptive optics system with a tunable narrow-band filter since AGNs are red shifted. Given that this combination is not currently available on the VLT, the AGN sample in near-future studies must be limited to those nearby.

Note added in proofs: The $3.3 \mu \mathrm{m}$ emission feature detected in our spectrum (Fig. 4) corresponds to the wavelength of the PAH $3.28 \mu \mathrm{m}$ emission feature. However, the equivalent width is found to be $1.2 \mathrm{~nm}$, about a hundred times less than the typical value for starburst dominated galaxies given by Imanishi (2002). Furthermore, studies of PAH emission toward a wide range of galactic sources with various densities and radiation fields indicate that the width of the PAH $3.28 \mu \mathrm{m}$ emission feature is invariant with a value $(F W H M)$ of $0.04 \mu \mathrm{m}$ (Verstraete 2001); Pierre Cox \& François Boulanger, private communication). Therefore our detected $3.3 \mu \mathrm{m}$ emission feature remains ambiguous. Anyway, the starburst activity has a negligible contribution to the total infrared luminosity.

Acknowledgements. These observations have been made in service mode at the VLT UT1 telescope. The authors want to thank the support astronomers who made the observations, in particular Nancy Ageorges, Vanessa Doublier and Christopher Lidman. The authors are also grateful to the anonymous referee for giving very useful comments on the manuscript.

\section{References}

Alexander, T., Lutz, D., Sturm, E., et al. 2000, ApJ, 536, 710

Alloin, D., Galliano, E., Cuby, J. G., et al. 2001, A\&A, 369, L33

Alonso-Herrero, A., Quillen, A. C., Simpson, C., Efstathiou, A., \& Ward, M. J. 2001, AJ, 121, 1369

Antonucci, R. 1993, ARA\&A, 31, 473

Barvainis, R. 1987, ApJ, 320, 537

Bottinelli, L., Gouguenheim, L., Fouque, P., \& Paturel, G. 1990, A\&AS, 82, 391

Braatz, J. A., Wilson, A. S., Gezari, D. Y., Varosi, F., \& Beichman, C. A. 1993, ApJ, 409, L5

Bridger, A., Wright, G. S., \& Geballe, T. R. 1994, in Infrared Astronomy with Arrays, The Next Generation, ed. Ian S. McLean, Astrophysics and Space Science Library, 190, 537

Cardelli, J. A., Clayton, G. C., \& Mathis, J. S. 1989, ApJ, 345, 245

Cid Fernandes, R., \& Terlevich, R. 1995, MNRAS, 272, 423

Clavel, J., Wamsteker, W., \& Glass, I. S. 1989, ApJ, 337, 236

Davies, R. I., Hajime, H., \& Ward, M. J. 1998, MNRAS, 300, 388

Gordon, K. D., Clayton, G. C., Witt, A. N., \& Misselt, K. A. 2000, ApJ, 533, 236

Imanishi, M., Terada, H., Sugiyama, K., et al. 1997, PASJ, 49, 69

Imanishi, M. 2000a, MNRAS, 319, 331

Imanishi, M. 2000b, MNRAS, 313, 165

Imanishi, M. 2001, AJ, 121, 1927

Imanishi, M. 2002, ApJ, 569, 44

Krolik, J. H. 1999, Active Galactic Nuclei: From the central black hole to the galactic environment (Princeton University Press, Princeton)

Lacombe, F., Marco, O., Geoffray, H., et al. 1998, PASP, 110, 1087

Marco, O. 1997, Ph.D. Thesis, Paris-Meudon Observatory \& University of Paris 6

Marco, O., Alloin, D., \& Beuzit, J. L. 1997, A\&A, 320, 399

Marco, O., \& Alloin, D. 1998, A\&A, 336, 823

Marco, O., \& Alloin, D. 2000, A\&A, 353, 465

Marco, O., \& Alloin, D. 2001, in AGN in their Cosmic Environment, EAS Publications Series, Vol. 1, 2001, ed. B. Rocca-Volmerange, \& H. Sol, JENAM 99, Toulouse, France, September 7-9, 1999, p. 11

Matt, G., Guainazzi, M., Frontera, F., et al. 1997, A\&A, 325, L13

Miles, J. W., Houck, J. R., \& Hayward, T. L. 1994, ApJ, 425, L37

Mouri, H., Kawara, K., Taniguchi, Y., \& Nishida, M. 1990, ApJ, 356, L39

Pendleton, Y. J., Sandford, S. A., Allamandola, L. J., Tielens, A. G., \& Sellgren, K. 1994, ApJ, 437, 683

Roche, P. F., Aitken, D. K., Smith, C. H., \& Ward, M. J. 1991, MNRAS, 248, 606

Rouan, D., Rigaut, F., Alloin, D., et al. 1998, A\&A, 339, 687

Thatte, N., Quirrenbach, A., Genzel, R., Maiolino, R., \& Tecza, M. 1997, ApJ, 490, 238

Tomono, D., Doi, Y., Usuda, T., \& Nishimura, T. 2001, ApJ, 557, 637

Verstraete, L., Pech, C., Moutou, C., et al. 2001, A\&A, 372, 981

Voit, G. M. 1992, MNRAS, 258, 841 